\title{
Stability and Oscillatory Behavior in Active Control System with Delays
}

\author{
Chunhua Feng* and Yuming Wei \\ College of Mathematics and Statistics, Guangxi Normal University, Guilin, China, 541004 \\ *Corresponding author
}

\begin{abstract}
In this paper, a model of active control system with delays is studied. Some sufficient conditions to guarantee the stability and oscillations for the system are established. Computer simulation is given to support our result.
\end{abstract}

Keywords-active control system; time delay; stability; oscillation

\section{INTRODUCTION}

In 1972, Yao proposed the concept of structural control [1]. Since then a lot of works have been done in the field of the active control structures. For example, Rohman and Nayfeh have studied active control of nonlinear oscillations in bridges [2]. Soong has investigated active control in civil engineering [3]. $\mathrm{Hu}$ and Wang have discussed the dynamics of controlled mechanical systems [4].

It is known that time delay is inevitable in active control systems. Therefore, many researchers have considered the effect of time delay in the control systems. Hong and Hughes have investigated the effect of time delay of the stability of flexible structure with rate feedback control [5]. Agrawal et al studied the instability due to time delay and its compensation in active control of structure [6,7]. Udwadia et al have investigated time delayed control design for active control of structure [8-11]. Recently, Peng et al have discussed an active control system with time delay feedback as follows [12]:

$$
m x^{\prime \prime}(t)+c x^{\prime}(t)+k x(t)=u x\left(t-\tau_{1}\right)+v x^{\prime\left(t-\tau_{2}\right)}+f(t)
$$

where $m$ is the mass, $c$ and $k$ are the damping and the stiffness, respectively, time delay $\tau_{1}$ is in the relative displacement feedback loop and time delay $\tau_{2}$ is in the relative velocity feedback loop, the external excitation is represented by $f(t)$. The non-dimension form of Eq. (1) could be written as

$$
x^{\prime \prime}(t)+2 \gamma x^{\prime}(t)+x(t)+g_{u} x\left(t-\tau_{1}\right)+g_{v} x\left(t-\tau_{2}\right)=\tilde{f}(t)
$$

$$
\text { where } \gamma=\frac{c}{2 m \sqrt{\frac{m}{k}}}, g_{u}=\frac{u}{k}, g_{v}=\frac{v}{2 m \sqrt{\frac{m}{k}}} \text {. }
$$

Let $\tau_{1}=\tau_{2}=\tau, \tilde{f}(t)=\beta x^{3}(t-\tau)$, the authors have studied the Hopf bifurcation and Fold bifurcation for system (2) by the linear stability analysis, respectively. Applying normal form method and center manifold theorem, the authors also integrated the direction of the bifurcations and the stability of periodic solution in the case $\tau_{1}=\tau_{2}=\tau$.

In this paper, we are interested the following system:

$$
x^{\prime \prime}(t)+2 \gamma x^{\prime}(t)+x(t)+g_{u} x\left(t-\tau_{1}\right)+g_{v} x\left(t-\tau_{2}\right)=\beta x^{3}\left(t-\tau_{3}\right)
$$

where $\beta x^{3}\left(t-\tau_{3}\right)=\tilde{f}(t)$ and $\left(\tau_{1}, \tau_{2}, \tau_{3}\right)$ may be different numbers. By mathematical analysis method, we study the stability and oscillation of the trivial solution of system (3).

\section{PRELIMINARIES}

System (3) can be written as

$$
\left\{\begin{array}{c}
x^{\prime}(t)=y(t) \\
y^{\prime}(t)=-x(t)-2 \gamma y(t)-g_{u} x\left(t-\tau_{1}\right)-g_{v} y\left(t-\tau_{2}\right)+\beta x^{3}\left(t-\tau_{3}\right)
\end{array}\right.
$$

The matrix form of system (4) is the following

$$
X^{\prime}(t)=A X(t)+B(t-\tau)+M_{\tau}(t)
$$

where $X(t-\tau)=\left(x\left(t-\tau_{1}\right), y\left(t-\tau_{2}\right)\right)^{T} . A=\left[\begin{array}{cc}0 & 1 \\ -1 & -2 \gamma\end{array}\right]$, $B=\left[\begin{array}{cc}-g_{u} & 0 \\ 0 & -g_{v}\end{array}\right], \quad M_{\tau}(t)=\left[\begin{array}{c}0 \\ \beta x^{2}\left(t-\tau_{3}\right)\end{array}\right]$. The linearized system of (5) is the following:

$$
X^{\prime}(t)=A X(t)+B(t-\tau)
$$

Let $\tau^{*}=\max \left\{\tau_{1}, \tau_{2}, \tau_{3}\right\}$ and $\tau_{*}=\min \left\{\tau_{1}, \tau_{2}, \tau_{3}\right\}$,Correspo nding to system (6) we consider two special cases 
(a) $\tau_{1}=\tau_{2}=\tau_{3}=\tau^{*}$, and $\quad X^{\prime}(t)=A X(t)+B\left(t-\tau^{*}\right)$

(b) $\tau_{1}=\tau_{2}=\tau_{3}=\tau_{*}$, and $X^{\prime}(t)=A X(t)+B\left(t-\tau_{*}\right)$

We adopt the following norms of vectors and matrices in this paper [13]:

$$
\|z(t)\|=\sum_{i=1}^{n}\left|z_{i}(t)\right|,\|A\|=\sum_{i=0}^{n}\left|a_{i j}\right|
$$

the measure $\mu(\mathrm{A})$ of a matrix $\mathrm{A}$ is defined by $\mu(\mathrm{A})$ $=\lim _{\theta \rightarrow 0^{+}} \frac{\|I+\theta A\|-1}{\theta}$, which for the chosen norms reduces to $\mu(\mathrm{A})=\max _{1 \leq j \leq n}\left(a_{i j}+\sum_{i=1, i \neq j}^{n}\left|a_{i j}\right|\right)$. A $>0$ (respectively, $\mathrm{A}<0$ ) which indicates that $\mathrm{A}$ is a positive (negative) definite matrix.

Lemma 1 If (i) $\beta>0, g_{u} \leq-1$ or (ii) $\beta<0, g_{u} \geq-1$. Then system (4) has a unique equilibrium. It is exactly the zero point.

Proof If $\left(x^{*}, y^{*}\right)^{T}$ is an equilibrium point of system (4), then $\left(x^{*}, y^{*}\right)^{T}$ satisfies the following equation:

$$
\left\{\begin{array}{c}
y^{*}=0 \\
-x-2 \gamma y^{*}-g_{u} x^{*}-g_{v} y^{*}+\beta^{* 3}=0
\end{array}\right.
$$

Thus, from (9) we have $y^{*}=0$ and

$$
\left(\beta x^{* 2}-\left(1+g_{u}\right)\right) x^{*}=0
$$

From (10), conditions (i) and (ii) guarantee that there exists one and only one real equilibrium point, it is exactly the zero point.

\section{MAIN RESUlts}

Theorem 1 Assume that system (5) has a unique equilibrium point. Let the eigenvalues of matrix be $\alpha_{i}(i=1,2)$. If $\operatorname{Re}\left(\alpha_{i}\right)<-a<0(i=1,2)$. Then the trivial solution of system (5) is asymptotically stable.

Proof According to the knowledge of time delay system, the stability of trivial solution of (7) implies the stability of trivial solution of (6). Noting that $\beta x^{3}$ is high order infinitesimal as $x \rightarrow 0$. Hence, if the trivial solution of (6) is asymptotically stable, then the trivial solution of system (5) is asymptotically stable. Thus the asymptotic stability of the trivial solution of system (7) implies the asymptotic stability of the trivial solution of system (5). Noting that each eigenvalue of matrix $B$ is zero. Since
$\operatorname{Re}\left(\alpha_{i}\right)<-a<0,(i=1,2)$, there exists $L \geq 11$ such that $e^{(A+B) t} \leq e^{-a t}$.Rewrite (7) in the form

$$
\begin{aligned}
& X^{\prime}(t)=(A+B) X(t)-B \int_{t-t^{*}}^{t} X^{\prime}(s) d s \\
& =(A+B) X(t)-B \int_{t-t^{*}}^{t}\left(A X(s)+B X\left(s-t^{*}\right)\right) d s, t \geq t^{*}
\end{aligned}
$$

So we get

$X(t)=e^{(A+B)\left(t-\tau^{*}\right)} X\left(\tau^{*}\right)-\int_{\tau^{*}}^{t} d s \int_{s-\tau^{*}}^{s} e^{(A+B)(t-s)} B\left(A X(u)+B X\left(u-\tau^{*}\right)\right) d u, t \geq \tau^{*}$

Hence for $t \geq \tau^{*}$ we have

$\|X(t)\| \leq K L e^{-a\left(t-\tau^{*}\right)}+L\|B\| \int_{\tau^{2}}^{t} d S \int_{s-\tau^{2}}^{s} e^{-a(t-s)}\|A\|\|X(u)\|+\|B\|\left\|X\left(u-\tau^{*}\right)\right\| d u, t \geq \tau^{*}$

For $t \geq \tau^{*}$ define

$\left.\|Y(t)\|=K L e^{-a\left(t-\tau^{*}\right)}+L\|B\| \int_{\tau^{*}}^{t} d s \int_{s-\tau^{-}}^{s} e^{-a(t-s)}\|A\|\|Y(u)\|+\|B\|\left\|Y\left(u-\tau^{*}\right)\right\|\right) d u, t \geq \tau^{*}$

Obviously, we have $|x(t)| \leq|y(t)|$ according to the comparison theorem of differential equations. We pointed out that there exists a positive constant $b(b<a)$ such that $Y(t)$ can be written as the form

$$
\|Y(t)\|=K L e^{-b\left(t-\tau^{*}\right)}, t \geq \tau^{*}
$$

Indeed,

$$
\begin{aligned}
& K L e^{-a\left(t-\tau^{*}\right)}+L\|B\| \int_{\tau^{*}}^{t} d s \int_{s-\tau^{2}}^{s} e^{-a(t-s)}\left(\|A\| \mid\|Y(u)\|+\|B\| Y Y\left(u-\tau^{*}\right) \|\right) d u \\
& =K L e^{-a\left(t-\tau^{*}\right)}+L\|B\| \int_{\tau^{*}}^{t} d s \int_{s-\tau^{*}}^{s} \cdot e^{-a(t-s)}\left(\|A\| K L e^{-b\left(t-\tau^{*}\right)}+\|B\| K L e^{-b\left(u-2 \tau^{*}\right)}\right) d u \\
& =K L e^{-a\left(t-\tau^{*}\right)}+L\|B\| \frac{K L\left(\|A\| e^{b \tau^{*}}+\|B\| e^{2 b t^{*}}\right)}{-b} \int_{\tau^{*}}^{t} \cdot e^{-a(t-s)}\left(e^{-b s}-e^{-b\left(s-\tau^{*}\right)}\right) d s \\
& =K L e^{-a\left(t-\tau^{*}\right)}+K L \frac{L\|B\|\left(\|A\| e^{b \tau^{*}}+\|B\| e^{2 b \tau^{*}}\right)}{b(a-b)} e^{-a t} e^{b \tau^{*}}\left(e^{(a-b) t}-e^{(a-b) \tau^{*}}\right)
\end{aligned}
$$

select a positive constant $b(b<a)$ such that

$$
\frac{L\|B\|(\|A\|+\|B\|)\left(e^{b \tau^{*}-1}\right)}{b(a-b)}=1
$$

Then

$$
\begin{aligned}
& K L e^{-a\left(t-\tau^{*}\right)}+K L \frac{L\|B\|\left(\|A\| e^{b \tau^{*}}+\|B\| e^{2 b \tau^{*}}\right)}{b(a-b)} e^{-a t} e^{b \tau^{*}}\left(e^{(a-b) t}-e^{(a-b) \tau^{*}}\right) \\
& =K L e^{-a\left(t-\tau^{*}\right)}+K L e^{-a t} e^{b \tau^{*}}\left(e^{(a-b) t}-e^{(a-b) \tau^{*}}\right)=K L e^{-b\left(t-\tau^{*}\right)}, t \geq \tau^{*}
\end{aligned}
$$


From (14), we immediately get $\|Y(t)\| \rightarrow 0$ as $t \rightarrow \infty$, implying that $\|X(t)\| \rightarrow 0$ as $t \rightarrow \infty$ This means that the trivial solution of system (5) is asymptotically stable.

Theorem 2 Suppose that system (5) has a unique equilibrium point. If $|\mu(A)| \leq\|B\| \mid$, then the trivial solution of system (5) is unstable, implying that system (5) has an oscillatory solution.

Proof Since $\beta x^{3}$ is high order infinitesimal as $x \rightarrow 0$.Therefore, if the trivial solution of (8) is unstable, then the trivial solution of system (5) is also unstable. Consider the system (8), the characteristicequation of (8) is the following:

$$
\operatorname{det}\left(\lambda I-A-B e^{-\lambda \tau_{*}}\right)=0
$$

We want to show that there is a positive eigenvalue of (18) under assumption. Consider function

$$
f(\lambda)=\lambda-\mu(A)-\|B\| e^{-\lambda \tau_{*}}
$$

where $f(\lambda)$ is a continuous function of $\lambda$. If $\mu(A)>0$, then we obviously have a $\lambda^{*}>0$ such that $f\left(\lambda^{*}\right)=0$ since $\|B\| e^{-\lambda^{*} \tau_{*}}>0$. If $\mu(A)<0$ then $f(0)=-u(A)-\|B\|<0$. Apparently there exists a suitably large $\lambda>0$ such that $f(\lambda)=\lambda-\mu(A)-\|B\| e^{-\lambda \tau_{*}}>0$. Therefore, there is a $\lambda^{* *} \in(0, \lambda)$ such that $f\left(\lambda^{* *}\right)=0$ by the continuity of $f(\lambda)$. Since there exists a positive eigenvalue of (18), the result follows. Again consider the following system:

$$
X^{\prime}(t)=A X(t)+B X\left(t-\tau^{*}\right)
$$

Similar to the above analysis, we know that there exists a positive eigenvalue of (20).

Now for each $\tau_{i}(i=1,2,3)$, satisfying $\tau_{*} \leq \tau_{i} \leq \tau^{*}$, the followingsystem

$$
X^{\prime}(t)=A X(t)+B X(t-\tau)
$$

has a positive eigenvalue, implying that the trivial solution of system (5) is unstable and system (5) has an oscillatory solution.

\section{Computer Simulation Result}

In system (3), we select parameters $\gamma=0.95$, $g_{u}=1.005, g_{v}=0.08, \beta=-0.0043$. Thus, system (3) has a unique equilibrium point. Obviously, the conditions of Theorem 1 are satisfied. When we fixed time delays $\tau_{1}=1.8, \tau_{2}=2.5, \tau_{3}=1.9$ and $\tau_{1}=2.8, \tau_{2}=4.5, \tau_{3}=3.8$ respectively, the trivial solution is convergent (Fig. 1a and 1b). When we select parameters $\gamma=1.28$, $g_{u}=1.15, \quad g_{v}=0.885, \quad \beta=-0.005$. The conditions of Theorem 1 are still hold. When we fixed time delays $\tau_{1}=6.8, \tau_{2}=5.5, \tau_{3}=4.8$, the trivial solution is convergent (Fig. 2a). However, when time delays are increased to $\tau_{1}=14.8, \tau_{2}=12.2, \tau_{3}=9.6$. respectively, an oscillatory solution is appeared (Fig. 2b). This implies that time delay induced oscillation. However, what are the critical time delay values between convergence and oscillation still is an open problem. We pointed out that the bifurcation method may be hard to deal with the case that time delays $\tau_{1}, \tau_{2}, \tau_{3}$ are different positive numbers as our simulation.
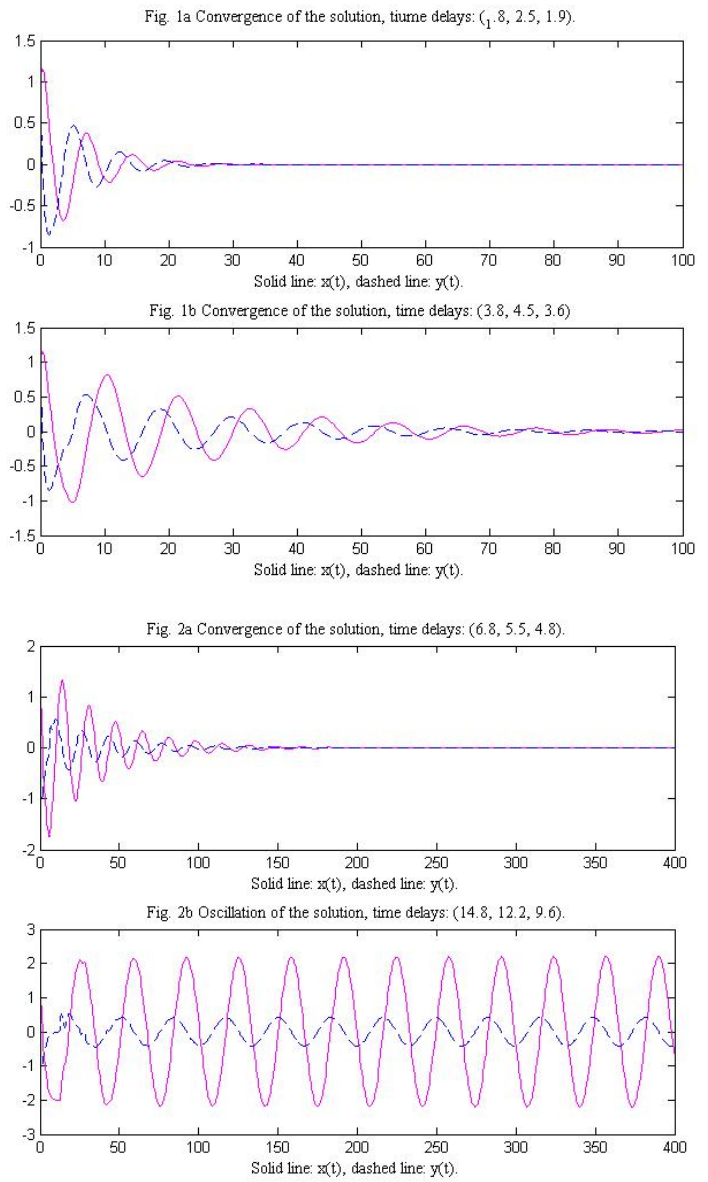

ACKNOWLEDGMENT

This work was partly supported by the National Natural Science Foundation of China (Grant No. 11361010) and the Guangxi Natural Science Foundation (Grant No. 2014GXNSFAA118002).

\section{REFERENCES}

[1] J.T.Yao, Concept of structural control, J. Struc. Div. ASCE 98 (1972) 1567-1574. 
[2] M.A. Rohman, A.H. Nayfeh, Active control of nonlinear oscillations in bridges, J. Eng. Mech. ASCE 113 (3) (1987) 335-348.

[3] T.T. Soong, State-of-the-art review: active structural control in civil engineering, Eng. Struct. 10 (1988) 74-84.

[4] H.Y. Hu, Z.H. Wang, Dynamics of controlled mechanical systems with delayed feedback, Springer-Verlag, Berlin, 2002.

[5] T. Hong, P.C. Hughes, Effect of time delay of the stability of flexible structure with rate feedback control, J. Vib. Control 7 (2001) 33-49.

[6] A.K. Agrawal, Y. Fujino, B.K. Bhartia, Instability due to time delay and its compensation in active control of structure, Earthquake Eng. Struc. Dyn. 22 (1993) 211-224.

[7] A.K. Agrawal, J.N. Yang, Effect of fixed time delay on stability and performance of active controlled civil engineering structures, Earthquake Eng. Struc. Dyn. 26 (1997)1169-1185.

[8] F.E. Udwadia, R. Kumar, Time-delayed control of classically damped structural systems, Int. J. Control 60 (5) (1994) 687-713.

[9] F.E. Udwadia, H. Bremen, R. Kumar, M. Hossienei, Time delayed control of structures systems, Earthquake Eng. Struc. Dyn. 32 (2003) 495-535.

[10] F.E. Udwadia, P. Phohomsiri, Active control of structures using time delayed positive feedback proportional control design, Struc. Control Health Monit. 13 (1) (2006) 536-552.

[11] F.E. Udwadia, H. Bremen, P. Phohomsiri, Time delayed control design for active control of structures: principles and applications, Struc. Control Health Monit. 14 (1) (2007) 27-61.

[12] J. Peng, L.H. Wang, Y.Y. Zhao, Y.B.Zhao, Bifurcation analysis in active control system with time delay feedback, Appl. Math. Comput. 219 (2013) 10073-10081.

[13] K. Gopalsamy, Stability and oscillations in delay differential equations of population dynamics, Kluwer Academic Publishers, Boston, 1992. 